\title{
A Study of Aerodynamic Characteristics of an Anti-tank Missile
}

\author{
Chun-Chi Li, Wei-Chan Hong
}

\begin{abstract}
The present study applied computational fluid dynamics to a type of contemporary antitank missile capable of entering into a unique top-attack mode, and explored the differences in the aerodynamic properties of the missile with its tailfins swinging at various angles. Simulation results indicated that the drag coefficient increased with the angle of attack, the swing angle of the vertical tailfins, and the negative swing angle of the horizontal tailfins. The lifting effect from the swinging of the vertical tailfins was observed to contribute $0.5 \%$ of the total lift, and the horizontal tailfins provided a higher lift when positioned in negative swing angles. As the angle of attack was increased, the lift coefficient also increased, and could be presented in a linear function involving the angle of attack and the swing angle of the tailfins: $\mathrm{C}_{\mathrm{L}}=\Phi(\alpha, \delta \mathrm{h})$. When $\alpha=5^{\circ}, \delta \mathrm{v}=0^{\circ}$, and $\delta \mathrm{h}$ $=0^{\circ}$ to $-10^{\circ}$, the lift-to-drag ratio of the missile $\left(\mathrm{C}_{\mathrm{L}} / \mathrm{C}_{\mathrm{D}}\right)$ was approximately 3 . The eight wings in the middle section of the missile contributed to approximately $64.5 \%$ of the total lift, implying that an adequate number of middle fins provide higher lift. When $\delta \mathrm{v}=0^{\circ}$ and $\delta \mathrm{h}=0^{\circ}$, the stalling angle of attack of the missile was $40^{\circ}$. The present study demonstrated from the aerodynamic properties of the missile that the missile had a high lift-to-drag ratio and excellent flight stability, enabling it to scramble rapidly then dive in a top-attack mode.
\end{abstract}

Keywords - computational fluid dynamics, anti-tank missile, Mach number, drag coefficient, lift coefficient, stalling angle of attack

\section{INTRODUCTION}

A guide and control system significantly increases the hit rate of anti-tank missiles. Combined with armor-piercing high-explosive warheads, the design effect of a single missile destroying a tank can be achieved. Current anti-tank missiles include the U.S. FGM-148 Javelin, AGM-114 Hellfire, FGM-77 Dragon, and BGM-71. The precision and destructiveness of these missiles characterize them as the greatest threat to tanks. The hardware composition of anti-tank missile systems is generally divided into four sections: the guidance, warhead, actuator, and propulsion sections. The exterior design of the missile body emphasizes aerodynamic characteristics in addition to structural strength. During missile flight, the corresponding aerodynamics, such as the lift, drag, and moment that are produced from the air flow against the surface of the missile body, directly influence its flight attitude, stability, and handling or operational characteristics. Therefore,

Chun-Chi Li, Department of Mechanical and Aerospace Engineering, Chung Cheng Institute of Technology / National Defense University, Taiwan, ROC. Email id: davidli560607@gmail.com

Wei-Chan Hong, Master Program of Mechanical Engineering, Chung Cheng Institute of Technology / National Defense University, Taiwan, ROC missiles with different functions involve.

Analysis methods for determining the aerodynamic parameters of flying objects include aerodynamic parameter values measured by conducting wind tunnel experiments and semi-empirical methods similar to those of the U.S. Air Force Missile DATCOM. The calculation efficiency of computers has significantly increased with the development of contemporary computer technology; therefore, computational fluid dynamics (CFD) simulation has gradually become the focus of aerodynamic calculations, and can facilitate wind tunnel experiments and subsequently decrease research costs. Tuncer et al. [1] conducted numerical simulations involving subsonic velocity missile bodies of different canard-tail aerodynamic distributions. The experiments examined an angle of attack $\alpha$ ranging from $0^{\circ}$ to $60^{\circ}$, calculated the relationship among $\alpha$, normal force coefficient $\mathrm{C}_{\mathrm{N}}$, and pitching moment coefficient $\mathrm{C}_{\mathrm{M}}$, and observed the location in which airflow creates separation, reconnection, and vortex phenomena. Champigny [2] examined the locations in which the boundary layer, vortex, and air separations occur because of the air viscosity effect, and selected four turbulence models to conduct a numerical simulation involving missile bodies with fins. Among these models, the Spalart-Allmaras turbulence model has undergone long-term empirical verifications, and is adaptable to the majority of existing complex flow fields, particularly to rise and drag problems of missile bodies with fins. DeSpirito [3] adopted the CFD numerical simulation and selected the Spalart-Allmaras turbulence model to calculate the aerodynamic effects of various $\alpha$ for missile bodies configured with and without fins. DeSpirito subsequently compared the results to that of the wind tunnel experiment, and observed an error within $5 \%$.

This study calculated the aerodynamics of an existing and active 3D anti-tank missile (Fig. 1) to examine the aerodynamic characteristics of a missile body at different flight attitudes. The missile has a unique top-attack mode that enables it to scramble to a considerable height in a relatively short distance and then dive on the thinnest armored spot of a tank. The eight middle wings are also worthy of note, because this is the highest number seen on contemporary antitank missiles. This feature was of interest to the present author, particularly for its aerodynamic properties and whether it was purposefully designed for the top-attack mode.

According to the data of Harris and Slegers [4] regarding this missile, when the flight trajectory adopts a top-attack model, the range is approximately $2,000 \mathrm{~m}$ and the flight time is approximately $16 \mathrm{~s}$. The velocity of the missile after detaching 
from the launch tube is $13 \mathrm{~m} / \mathrm{s}$, and the pitch angle is $\theta=18^{\circ}$. Harris and Slegers provided five performance curve graphs, respectively presenting the height-length plot ( $\mathrm{H}-\mathrm{L}$ plot), flight velocity-length plot (V-L plot), $\alpha$-time plot ( $\alpha$-t plot), $\theta$-time plot $(\theta-\mathrm{t}$ plot $)$, and the control tail fin deflection-time $(\delta-\mathrm{t})$ plot. Analysis was conducted on the information provided by Harris and Slegers, and it was discovered that the power of the missile regarding velocity originates from the engine at the rear of the missile body. The mass of the solid propellant in the engine is a constant value, and limits the basic flight range or length and velocity. Regardless of any attack model or ballistic trajectory, the flight velocity is approximately between 0.3 and $0.5 \mathrm{M}$. Regarding the flight attitude, the period between the missile's detachment from the launch tube and the activation of the guide and control system is the preliminary trajectory. This period is uncontrolled and, thus, exhibits inferior stability. After the guide and control system is activated, the trajectory is immediately directed toward the default trajectory, subsequently requiring greater control. The primary attitude control originates from the jet vane thrust vector system of the engine. Substantial trajectory corrections during this period result in a significant variation in $\alpha$. When the engine has completed functioning and the missile body has entered the default trajectory, the subsequent attitude correction is controlled by the four control fins on the rear of the missile body. The tail fins continuously correct the trajectory path or attitude during stable flight. Data show that unless lift is required, the control system primarily maintains the flight attitude of the missile body at a low $\alpha$ to decrease the negative effects caused by air resistance or drag. Therefore, the $\alpha$ of the missile during stable flight is maintained between $0^{\circ}$ and $5^{\circ}$. The four swept-back control tail fins of the missile are designed to be cross-shaped, and lift, drag, and lateral force are correspondingly produced during flight based on the deflection and swing of the control tail fin. Rolling moment, pitching moment, and yawing moment are produced in correspondence to the center of pressure $(\mathrm{CP})$. These three types of moment can alter the flight attitude of the missile body. Variations in the tail fin deflection angle directly affect the air flow field, resulting in different aerodynamic effects; therefore, the above factors must be considered during calculation and simulation. The tail fin comprises the Y-directional vertical tail fin and the Z-directional horizontal tail fin. Based on the $\delta$-t curve of the control tail's deflection angle in [5], the deflection angle of the vertical and horizontal tails are approximately controlled between $-10^{\circ}$ to $10^{\circ}$. This study defined the deflection angle of the vertical tail fin as $\delta_{\mathrm{v}}$ and that of the horizontal tail fin as $\delta_{\mathrm{h}}$. Counterclockwise rotation indicates a positive value, as shown in Fig. 2.

Regarding literature on wind tunnel experiments for this anti-tank missile, Lei et al. [5] conducted a series of wind tunnel experiments on six types of missile fin distributions with an Mach numbers between 0.3 and 0.5 . The results indicated that a distribution of eight mid-fins and four tail fins exhibited superior stability in the subsonic velocity range of the experiment. In addition, a $0.4-\mathrm{M}$ experiment was conducted at $\alpha$ $=0^{\circ}$, and Lei et al. acquired a drag coefficient of $\mathrm{C}_{\mathrm{D}}=$ 0.291.Based on these results, this study employed the following flight criteria for anti-tank missiles: $\mathrm{M}=0.3 \sim 0.5, \alpha=0^{\circ} \sim 5^{\circ}, \delta_{\mathrm{v}}=$ $0^{\circ} \sim 10^{\circ}$, and $\delta_{\mathrm{h}}=-10^{\circ} \sim 10^{\circ}$. These criteria were used to examine the aerodynamic characteristics of missiles. Relevant information is further provided as reference for future development of novel anti-tank missiles.

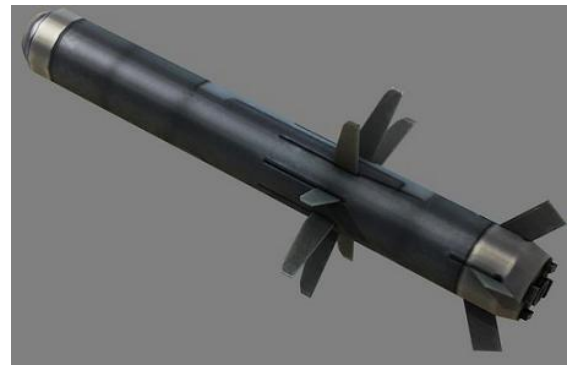

Fig1. Anti-tank missiles of this study[1]

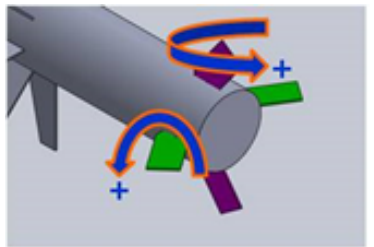

Fig 2. The control tail fin swing schematic

\section{II.PROBLEMS AND METHODS}

\section{A. Governing Equation and Numerical Methods}

The governing equations are the Reynolds averaged Navier-Stokes equations, the conservation can be expressed as follows

$$
\frac{\partial U}{\partial t}+\frac{\partial F}{\partial x}+\frac{\partial G}{\partial y}+\frac{\partial H}{\partial z}=\frac{\partial F_{v}}{\partial x}+\frac{\partial G_{v}}{\partial y}+\frac{\partial H_{v}}{\partial z}
$$

In solving equation (1), convection terms $(\mathrm{F}, \mathrm{G}, \mathrm{H})$ are calculated by AUSM+ scheme, while viscosity and diffusion flux terms $\left(\mathrm{F}_{v}, \mathrm{G}_{v}, \mathrm{H}_{v}\right)$ are calculated using the central difference method. Discrete space terms are to form a group of ordinary differential equations followed by time integration to obtain the numerical solution. Turbulence model adopted the Spalart-Allmars equation. The condition of inlet boundary and outlet boundary should be set at pressure-far-field condition and pressure-outlet condition, respectively.

\section{B. Grid System and Numerical Code Validation}

This study set the computational domain inlet as one times the length of the missile extended from the tip of the missile, and the distance between the rear of the missile body and the computational domain outlet as 2.5 times the length of the missile. The surface grid and various parts of the missile body are identified in Fig. 3a. The cylinder diameter was two times the length of the missile, and the computational domain is shown in Fig. 3b. To verify the accuracy of the numerical simulation, this study employed the wind tunnel experiment data used in [5] as comparative data for simulation verification. 
When $\mathrm{M}=0.4$ and $\alpha=0^{\circ}$, the missile body's $\delta_{\mathrm{v}}=0^{\circ}$ and $\delta_{\mathrm{h}}=0^{\circ}$, and experimental $\mathrm{C}_{\mathrm{D}}=0.291$. Regarding lattice point calculations, the concentration of lattice points were increased on the surface of the missile body wall, between the mid-fin and the tail fin, at the boundary layer, and in the rear vortex area at the bottom of the missile. Nine grid number systems were used to conduct program verification. The calculation results were similar to the experimental drag coefficients, as shown in Fig. 4. For superior calculation efficiency, two million grids were used as the grid system for simulation.

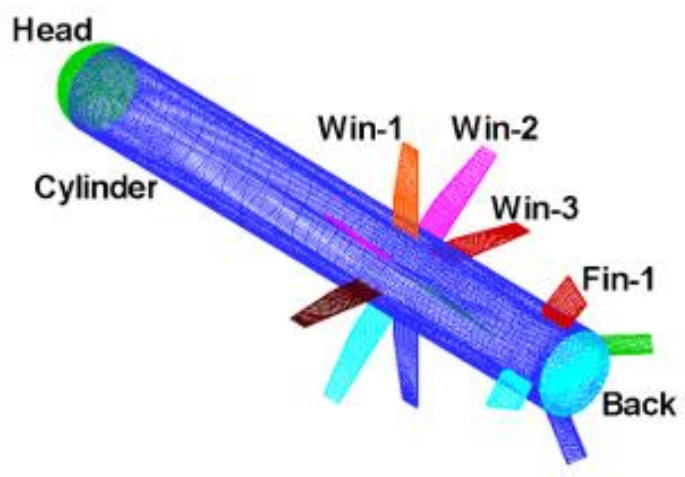

Fig 3a. Anti-tank grid diagram

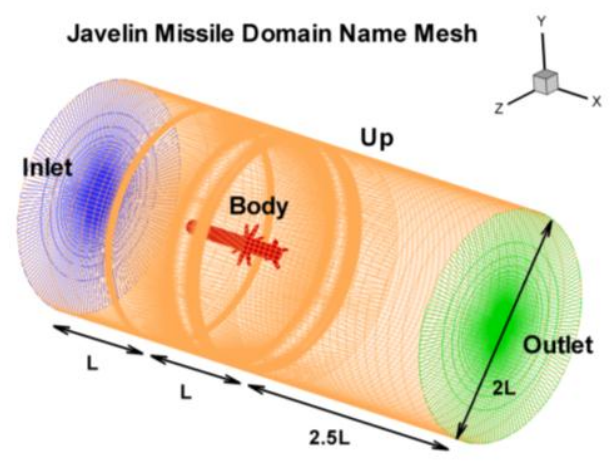

Fig 3b. Schematic diagram of computational domain

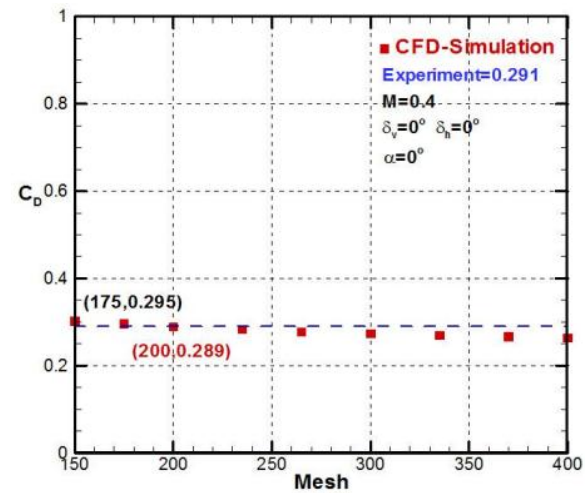

Fig 4. The drag coefficient comparison verify the independence of the grid

\section{Model Parameter Explanation}

The various simulation parameters for the calculation model are explained as follows: The order of arrangement is $M, \alpha, \delta_{v}$, and $\delta_{\mathrm{h}}$. For example, 3_5_0_10 indicates the flight condition under $\mathrm{M}=0.3, \alpha=5^{\circ}, \delta_{\mathrm{v}}=0^{\circ}$, and $\delta_{\mathrm{h}}=10^{\circ}$; and 3_5_0_"-10 10" denotes that $\mathrm{M}=0.3, \alpha=5^{\circ}$, and $\delta_{\mathrm{v}}=0^{\circ}$, where $\delta_{\mathrm{h}}$ swings from $-10^{\circ}$ to $10^{\circ}$. The origin location is at the tip of the missile. This study divided the upper and lower sections of the missile body into four primary sections with the axis and mid-fin as a benchmark (Fig. 5). The upper section of the missile body is the leeward side, which was further divided into Sections I and II; and the bottom section of the missile body is the windward side, which was further divided into Sections III and IV.

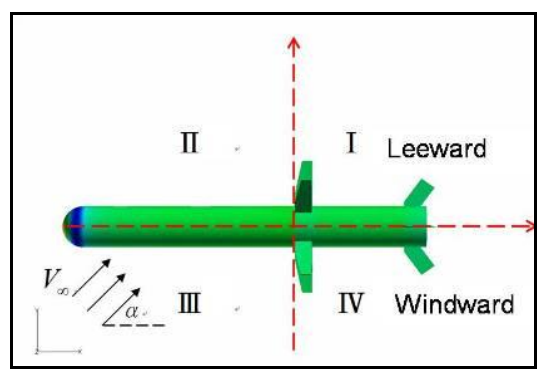

Fig 5. The anti-tank missile partition schematic diagram

\section{RESULTS AND DISCUSSION}

\section{A. An Examination of the Drag Coefficient}

When $\mathrm{M}$ and $\delta_{\mathrm{v}}$ are fixed, $\delta_{\mathrm{h}}$ is adjusted as $-10^{\circ},-5^{\circ}, 0^{\circ}, 5^{\circ}$, and $10^{\circ}$ for simulation. The results show that the greater the angle is, the greater the $C_{D}$. When $\alpha=0^{\circ}, C_{D}$ is the same when the positive and negative $\delta_{\mathrm{h}}$ are identical, and the representation in the figure is subsequently symmetrical (Fig. 6). When $\alpha$ is positive, and the positive and negative $\delta_{\mathrm{h}}$ have identical angles, a negative $\delta_{\mathrm{h}}$ possesses a greater drag coefficient than a positive $\delta_{\mathrm{h}}$ does. Furthermore, the greater the angle is, the greater the $\mathrm{C}_{\mathrm{D}}$, as shown in Fig. 7. Fig. 8 shows the calculation results of 5_5_10_"-10 10". Regarding the pressure distribution on the surface of the missile body, when air flow enters Section I, air flow closer to the junction wall surface between the tail fin and the missile body is compressed, and the pressure in this section is subsequently increased. This indicates that a portion of the missile's kinetic energy is transformed into pressure, and subsequently produces drag. A different $\delta_{\mathrm{h}}$ also results in different air flow compression situations near the tail fin section, subsequently producing different pressure gradients. When $\delta_{\mathrm{h}}=$ $0^{\circ}, 5^{\circ}$, and $10^{\circ}$, air flow is more likely to flow smoothly past the surface of the tail fin wall; thus, the area affected by high-pressure is smaller. The maximum pressure is approximately 4,000 $\mathrm{Pa}$, and the area affected by high pressure significantly expands when $\delta_{\mathrm{h}}=-5^{\circ}$ and $-10^{\circ}$. When $\delta_{\mathrm{h}}=-5^{\circ}$, the maximum pressure on the conjoining wall surface of the tail fin and the surface of the missile body exceeds approximately $5,000 \mathrm{~Pa}$. When $\delta_{\mathrm{h}}=-10^{\circ}$, the maximum pressure exceeds 7,000 $\mathrm{Pa}$, indicating that more kinetic loss occurs when $\delta_{\mathrm{h}}=-10^{\circ}$, producing greater drag. 


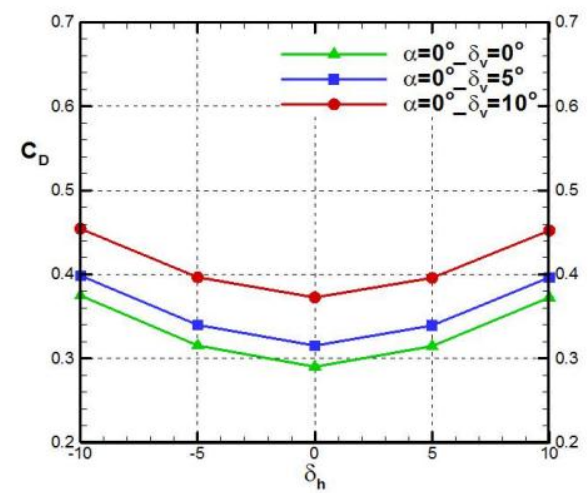

Fig 6. Drag coefficient plots $\left(\mathrm{M}=0.3, \alpha=0^{\circ}, \delta \mathrm{v}=0^{\circ} \sim 10^{\circ}, \delta \mathrm{h}=-10^{\circ} \sim 10^{\circ}\right)$

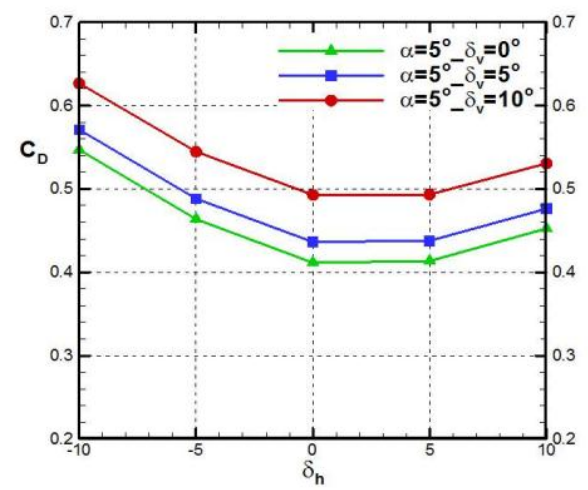

Fig 7. Drag coefficient plots $\left(\mathrm{M}=0.5, \alpha=5^{\circ}, \delta \mathrm{v}=0^{\circ} \sim 10^{\circ}, \delta \mathrm{h}=-10^{\circ} \sim 10^{\circ}\right)$
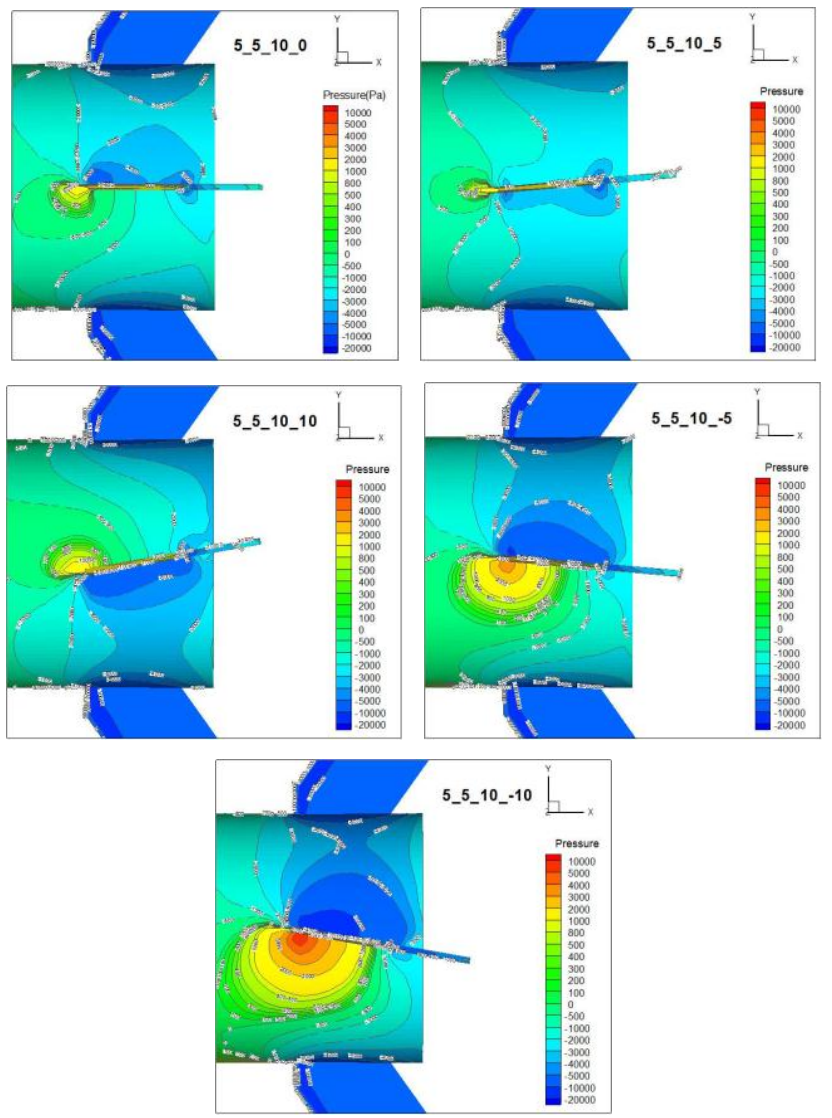

Fig 8 . The static pressure contour of the horizontal tail distribution (5_5_10_"-10 10")

\section{B. An Examination of Lift Coefficients}

Fig. 9 shows that when $\mathrm{M}=0.5$, the lift coefficient increases along with $\alpha$. When $\delta_{\mathrm{h}}$ is fixed, the swing angle or deviation for $\delta_{\mathrm{v}}$ possesses similar lift coefficients, and almost no influence is produced, because the $\delta_{\mathrm{v}}$ projection areas that are affected by lift show little change. The lift coefficient is influenced by $\delta_{\mathrm{h}}$; therefore, when the swing angle is between $-10^{\circ}$ and $10^{\circ}$, the lift coefficient undergoes a linear increase. The calculation results for 5_5_"0 10"_5 were selected, and an X-Y surface cross-sectional view of the flow field was conducted at $\mathrm{Z}=0$. The observations for the pressure distribution of the upper- and lower- sections of the missile body are shown in Fig. 10. The high-pressure section occurs more significantly at the windward side. With an $\alpha$ that does not equal $0^{\circ}$ occurs, air flow produces a high-pressure section close to the windward side of the mid-fin and a low-pressure section close to its leeward side, subsequently producing lift. In addition, the pressure distribution curves of these three flow fields are extremely approximate. Minor pressure distribution differences are only observed at the tail fin section. Fig. 11 shows the location on the missile body surface where the pressure data were retrieved. Fig. 12 shows the surface pressure distribution curves of the upperand lower-surfaces on the missile body. Significant surface pressure differences are observed near the head and the mid-fin of the missile. In addition, the pressure on the windward side of the missile body exceeds that of the leeward side. This pressure difference between the upper- and lower-sides results in an up-ward lifting effect of the missile body; that is, the lift. The surface pressure curve in Fig. 12 shows that, regarding the simulation results of 5_5_"0 10"_5, sections aside from or in front of the tail fin exhibit no upper- or lower-surface pressure curve distribution differences. Only minimal pressure changes occur at the tail fin section. Table I shows the calculation results of the missile lift for various components at 5_5_"0 10"_5. The lift effect caused by $\delta_{\mathrm{v}}$ accounts for only $0.5 \%$ of the influence on the total lift. Therefore, $\delta_{\mathrm{v}}$ has an insignificant influence on the lift coefficient.

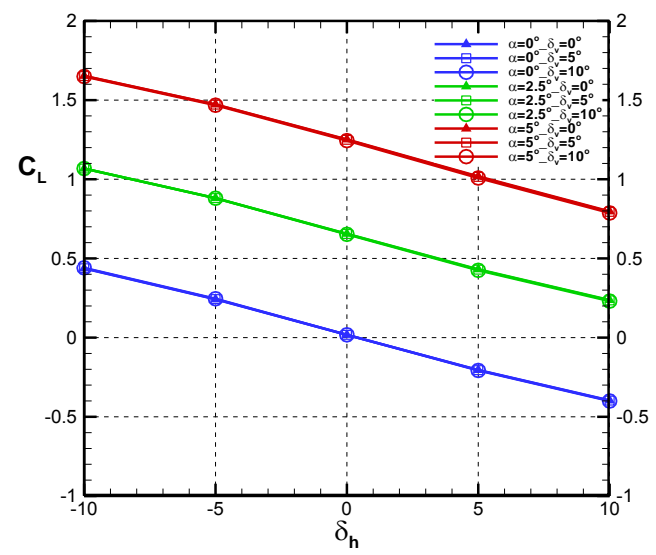

Fig 9. lift coefficient in $\mathrm{M}=0.5$ 

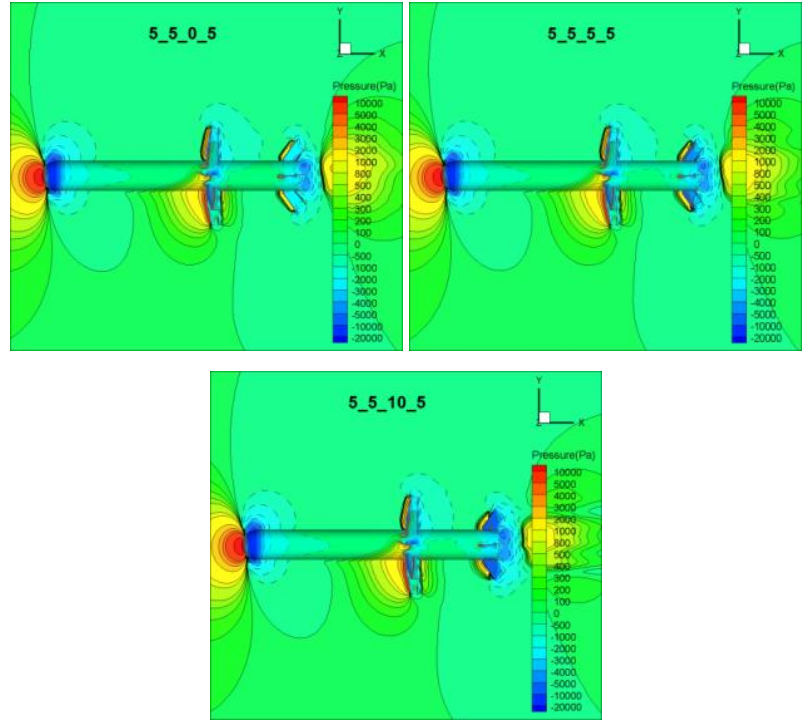

Fig 10. The static pressure contour (5_5_"0 10"_5)

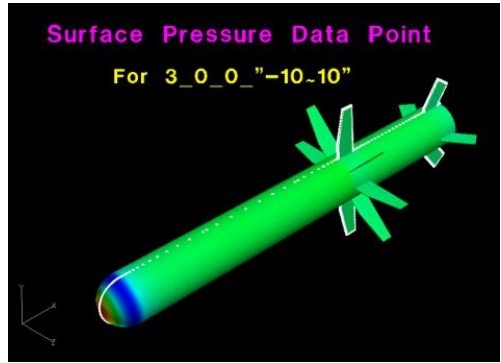

Fig 11.Schematic diagram of surface pressure data acquisition

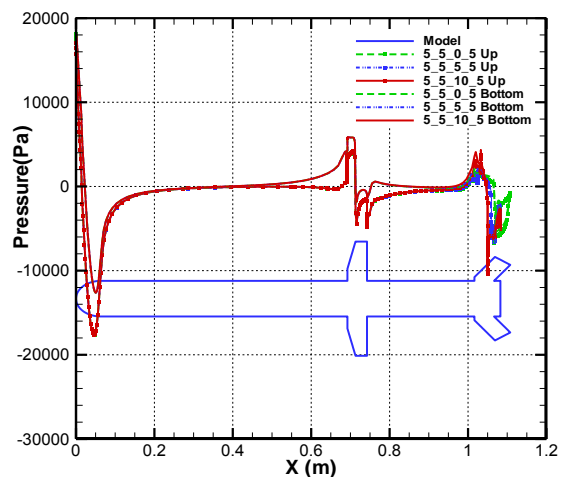

Fig 12. The static pressure contour of missile surface (5_5_"0 10"_5)

\begin{tabular}{|c|c|c|c|}
\hline Term & 5_5_0_5 & 5_5_5_5 & 5_5_10_5 \\
\hline Head & 29.2 & 29.2 & 29.19 \\
\hline Cylinder & 71.07 & 70.88 & 70.56 \\
\hline Back & -4.03 & -4.16 & -4.45 \\
\hline Win-1 & -0.39 & -0.39 & -0.4 \\
\hline Win-2 & 17.57 & 17.65 & 17.72 \\
\hline Win-3 & 35.87 & 35.82 & 35.76 \\
\hline Win-4 & 19.76 & 19.66 & 19.57 \\
\hline Win-5 & -0.02 & -0.02 & -0.02 \\
\hline Win-6 & 18.66 & 18.77 & 18.89 \\
\hline Win-7 & 36.03 & 36.06 & 36.07 \\
\hline Win-8 & 19.95 & 19.86 & 19.76 \\
\hline Fin-1 & -0.65 & -0.95 & -1.34 \\
\hline Fin-2 & -7.04 & -7.06 & -7.08 \\
\hline Fin-3 & 0.42 & 0.39 & -0.004 \\
\hline Fin-4 & -7.12 & -7.18 & -7.30 \\
\hline Net & 229.28 & 228.53 & 226.92 \\
\hline
\end{tabular}

\section{Stalling Angle of Attack}

Regarding the stalling angle of attack of the missile, an analysis was conducted to explore its relationship with the lift coefficient, specifically for the speeds of $0.3 \mathrm{~m}, 0.4 \mathrm{~m}$, and 0.5 $\mathrm{m}$ when the angle of attack $\alpha=0^{\circ}-50^{\circ}$ and the swing angles of both vertical $\left(\delta_{\mathrm{v}}\right)$ and horizontal $\left(\delta_{\mathrm{h}}\right)$ tailfins are $0^{\circ}$ (as shown in Fig. 13). The results revealed that in the three scenarios, stalling occurred at approximately $\alpha=40^{\circ}$. The curve of lift suggested that greater lift could be obtained through either a greater speed or a greater angle of attack. Differentiating lift with angle of attack $\left(\mathrm{dF}_{\mathrm{L}} / \mathrm{d} \alpha\right)$ achieved the curve of lift derivative illustrated in Fig. 14, from which the derivative $\left(\mathrm{dF}_{\mathrm{L}} / \mathrm{d} \alpha\right)$ was observed to diminish with the increase in angle of attack. This indicated that in a higher angle of attack, particularly when $\alpha=40^{\circ}$, the lift reached its maximum. Contrarily, when the angle of attack exceeded $40^{\circ},\left(\mathrm{dF}_{\mathrm{L}} / \mathrm{d} \alpha\right)<0$ and the lift started to decrease. Hence, the ideal stalling angle of attack of the antitank missile was determined to be $40^{\circ}$.

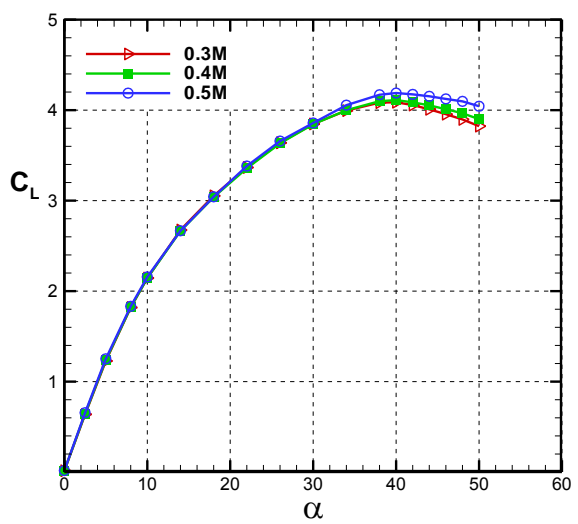

Fig 13.The plots of the lift coefficients with attack angles

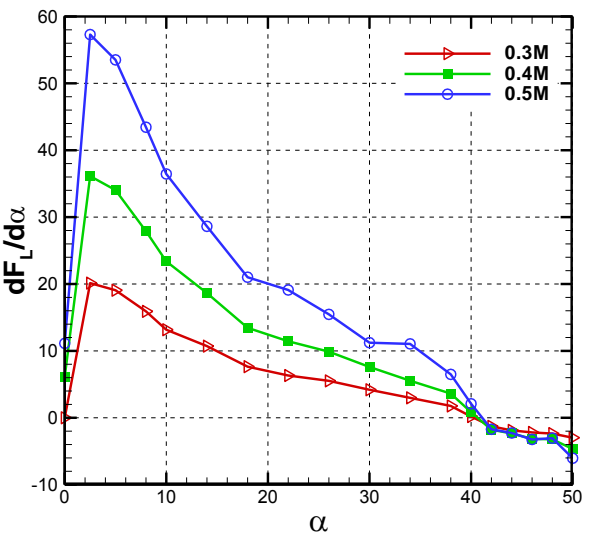

Fig 14.The plots of differentiating lift with angle of attack

\section{CONCLUSIONS}

Based on the previous content, this study presents the following conclusions. When $\alpha=0^{\circ}$ and $\mathrm{M}$ and $\delta_{\mathrm{v}}$ are fixed, adjusting $\delta_{\mathrm{h}}$ to a greater angle produces a greater drag coefficient. In addition, the drag coefficients are identical when the positive and negative angles are identical. When $\alpha$ is positive, a negative $\delta_{\mathrm{h}}$ produces a greater drag coefficient than that of a positive $\delta_{\mathrm{h}}$. In addition, a greater $\delta_{\mathrm{h}}$ results in a greater drag coefficient. Therefore, when conducting missile body 
flight attitude control, turning the horizontal tail fin toward a negative swing angle produces greater drag. When $\delta_{\mathrm{v}}$ and $\alpha$ are fixed, a greater lift is produced at $\delta_{\mathrm{h}}=-10^{\circ}$. When $\delta_{\mathrm{h}}=-10^{\circ}$ swings to a positive angle, the lift coefficient exhibits a linear decrease. When $\alpha=0^{\circ}$ and $\delta_{\mathrm{h}}>0^{\circ}$, negative lift is produced, subsequently resulting in a decrease in the missile body's flight height. Therefore, when lift is required, $\delta_{\mathrm{h}}$ should be adjusted to a negative angle, because the greater the negative swing angle is, the greater the lift. Furthermore, the influence of the $\delta_{v}$ on lift is insignificant, accounting for only $0.5 \%$ of the influence on the total lift. When $\alpha$ and $\delta_{\mathrm{h}}$ are identical, their lift coefficients are also identical. Lift coefficients can be perceived as a linear function created by $\alpha$ and $\delta_{\mathrm{h}}$; that is, $\mathrm{C}_{\mathrm{L}}=\Phi(\alpha, \delta \mathrm{h})$. When $\alpha=5^{\circ}$, $\delta_{\mathrm{v}}=0^{\circ}$, and $\delta_{\mathrm{h}}=0^{\circ}$ to $-10^{\circ}$, the lift-to-drag ratio of the missile $\left(\mathrm{C}_{\mathrm{L}} / \mathrm{C}_{\mathrm{D}}\right)$ was approximately 3 . The eight fins in the middle section of the missile contributed to approximately $64.5 \%$ of the total lift, implying that an adequate number of middle fins provide higher lift. When $\delta_{\mathrm{v}}=0^{\circ}$ and $\delta_{\mathrm{h}}=0^{\circ}$, the stalling angle of attack of the missile was $40^{\circ}$. The present study demonstrated from the aerodynamic properties of the missile that the missile had a high lift-to-drag ratio and excellent flight stability, enabling it to scramble rapidly then dive in a top-attack mode.

\section{ACKNOWLEDGMENT}

The author would like to thank the Ministry of Science and Technology of the Republic of China for financially supporting this research under Contract No. MOST 103-2221-E-606-019-

\section{REFERENCES}

[1] I. H. Tuncer, M. F. Platzer, and R. D. VanDyken, "A computational study of a close-coupled delta canard-wing-body configuration," in $199614^{\text {th }}$ AIAA Applied Aerodynamics Conf., New Orleans, LA, 96-2400. https://doi.org/10.2514/6.1996-2440

[2] P. Champigny, P. d'Espiney, M. Bredif, H. Broussard, and J. Gillyboeuf, "Numerical simulation of vortex flows around missile configurations," in 2003 RTO AVT Symp. , Loen, Norway, pp. 1-13.

[3] J. DeSpirito, Base pressure computations of the DERA generic missile wind tunnel model. Army Research Laboratory, Maryland, U.S.A, pp.1-12. 2005

[4] J. Harris, and N. Slegers, "Performance of a fire-and-forget anti-tank missile with a damaged wing," Mathematical and Computer Modelling, vol. 50, pp. 292-305, 2009. https://doi.org/10.1016/j.mcm.2009.02.009

[5] J. M. Lei, and J. S. Wu, "An experiment investigation of the aerodynamic characteristic for a multi-wing light anti-tank missile," ACTA Armamentarii, vol. 26, no. 5, pp. 709-711, 2005.

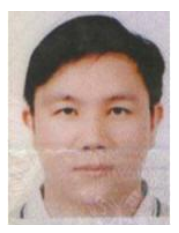

\section{Chun-Chi Li}

Associate professor, Department of Mechanical and Aerospace Engineering, CCIT, NDU, Tauyuan County, Taiwan, ROC, 2009-present. Research field in CFD, Heat and mass transfer, Porous media, Shock waves. 